\title{
Spontaneous shear flow in confined cellular nematics
}

\author{
G. Duclos ${ }^{\# 1}$, C. Blanch-Mercader ${ }^{\# 1}$, V. Yashunsky ${ }^{\# 1}$, G. Salbreux ${ }^{2}$, J.-F. Joanny ${ }^{1,3}$, J. \\ Prost $^{1,4,{ }^{*}, \text { P. Silberzan }}{ }^{1,}$ \\ ${ }^{1}$ Laboratoire PhysicoChimie Curie, Institut Curie, PSL Research University - Sorbonne \\ Universités, UPMC - CNRS. Equipe labellisée Ligue Contre le Cancer ; 75005, Paris, France \\ ${ }^{2}$ The Francis Crick Institute, London, UK \\ ${ }^{3} \mathrm{ESPCI}$ Paris, Paris, France \\ ${ }^{4}$ Mechanobiology Institute, National University of Singapore, Singapore \\ \# These authors contributed equally to this work.
}

\section{Abstract}

In embryonic development or tumor evolution, cells often migrate collectively within confining tracks defined by their microenvironment 1,2. In some of these situations, the displacements within a cell strand are antiparallel 3, giving rise to shear flows. However, the mechanisms underlying these spontaneous flows remain poorly understood. Here, we show that an ensemble of spindle-shaped cells plated in a well-defined stripe spontaneously develop a shear flow whose characteristics depend on the width of the stripe. On wide stripes, the cells self-organize in a nematic phase with a director at a well-defined angle with the stripe's direction, and develop a shear flow close to the stripe's edges. However, on stripes narrower than a critical width, the cells perfectly align with the stripe's direction and the net flow vanishes. A hydrodynamic active gel theory provides an understanding of these observations and identifies the transition between the non-flowing phase oriented along the stripe and the tilted phase exhibiting shear flow as a Freedericksz transition driven by the activity of the cells. This physical theory is grounded in the active nature of the cells and based on symmetries and conservation laws, providing a generic mechanism to interpret in vivo antiparallel cell displacements.

Collective cell migration is classically associated with adhesive cell-cell contacts that can ensure large velocity correlation lengths 3-12. However, cells lacking stable cell-cell adhesions such as fibroblasts have been shown to collectively orient in nematic phases 1316 and move in "streams" in dense monolayers. Interestingly, such streams have been reported in vivo in embryonic development 1 and cancer 2 . They are often accompanied by bidirectional flows of cells within the same strand. In particular, cancer cells migrating collectively in vivo in effective "channels" formed between collagen fibers have been

\footnotetext{
Users may view, print, copy, and download text and data-mine the content in such documents, for the purposes of academic research, subject always to the full Conditions of use:http://www.nature.com/authors/editorial_policies/license.html\#terms

*Jacques.prost@curie.fr; Pascal.silberzan@curie.fr.

Data availability

The data that support the findings of this study are available from the corresponding author upon reasonable request.
} 
observed to move away from but also toward the tumor they originate from 3. A similar feature has been observed in the migration of neural precursors in development 17,18. In vitro, when confined at high density in adhesive tracks, some cell types perfectly align in the main direction of the track and progressively stop moving 13 , while others orient with a finite angle relative to the local direction of the pattern and migrate in streams 19-21.

In the present letter we investigate the orientation and the dynamics of elongated Retinal Pigment Epithelial (RPE1) cells and C2C12 mouse myoblasts within confining stripes and analyze their behavior within the framework of a hydrodynamic active gel theory.

RPE1 cells in dense monolayers organize together in an active nematic phase. To study their behavior under confinement, we micropatterned glass substrates to define adhesive stripes of width $L(10 \mu m<L<1400 \mu m) 13,22$. In the first $30 \mathrm{~h}$ after confluence, cells build a dense monolayer. Between $30 \mathrm{~h}$ and $70 \mathrm{~h}$ they also dynamically develop an unstructured second layer on top of the first one, keeping displacements and orientations in register. Therefore, we considered the system as quasi-bidimensional up to $50 \mathrm{~h}$ after confluence.

When confined in a $500 \mu \mathrm{m}$-wide stripe, RPE1 cells oriented perfectly together within $~ 30$ $\mathrm{h}$ by annihilation of the characteristic nematic disclination defects 16,21,23 (Supplementary Fig. 2). Their orientation made a finite angle with the direction of the stripe (Figure 1A-E). In contrast, NIH-3T3 cells perfectly align with the stripe's direction for widths up to $500 \mu \mathrm{m}$ 13 (Supplementary Fig. 3A,B,G). Angles were averaged in the direction of the stripe to access the angle profile across its width $\theta(X)$ (Figure 1F). This tilt angle was larger at the edges $\left(\theta(x= \pm L / 2)=\theta_{M}=81^{\circ} \pm 19\right)$ and smaller at the center of the stripe $\left(\theta(x=0)=\theta_{m}=\right.$ $74^{\circ} \pm 29$. Of note, the sign of the orientation (right- or left-handed) of the cells relative to the stripe direction was very reproducible (Supplementary Fig. 4), meaning that this organization reflects an intrinsic tissue-scale chiral symmetry (handedness) as previously reported in ref. 19,24-26.

The velocity field in the confined cell layer was characterized by large fluctuations. However, averaging in space and time (Figure 1G) evidenced antiparallel flows of cells along the two edges of the stripe, amounting to a shear flow (Figure 1H, Supplementary Video 1). We also measured a cell flow in the transverse direction - $x$ - from the edges toward the center of the stripe (Figure 1I). The y-component of the velocity $v_{y}$ was significant over a distance $\lambda \approx 40 \mu \mathrm{m}$ next to the edges of the stripe and vanished in its center (Figure $1 \mathrm{~J}$ ). $\lambda$ is a friction screening length 16 . The $\mathrm{x}$-component $v_{X}$ was of smaller amplitude next to the edges but propagated further in the cell sheet (Figure $1 \mathrm{~K}$ ). Of notice, the shear flows were abolished in presence of the myosin inhibitor blebbistatin, confirming that they result directly from of cell activity. In contrast, blebbistatin didn't affect the convergent flows (Supplementary Fig. 5).

We then studied how these different quantities varied with the width of the stripe $L$ ( $10 \mu \mathrm{m}<$ $L<1400 \mu \mathrm{m}$, (Figure 2A-G)), considering only the fields of view free of topological defects. We found that the angle between the director and the stripe direction increased with width (Figure 2A-C) and that, after rescaling, the convergent flows profiles were universal (Figure $2 \mathrm{G})$. In contrast, the friction screening length was independent of the stripe width : $\lambda=44$ 
$\pm 10 \mu \mathrm{m}$ (Supplementary Fig. 6). This intrinsic screening length prevents a simple rescaling of the $v_{y}$ shear velocity profiles (Figure 2E). Strikingly, below a critical width $L_{\mathcal{c}}(40 \mu \mathrm{m}<$ $L_{c}<50 \mu \mathrm{m}$ ), the cells perfectly aligned with the main direction of the track (Figure 2A, $3 \mathrm{~A}, \mathrm{~B}, \mathrm{G})$ and the average shear flow vanished although the cells remained very motile and the convergent flow was not affected (Figure 3C,D,E,F,H,I). The shear velocity fluctuated in space and time about a zero mean (Figure 3E,I).

In summary, for $L>L_{\mathcal{C}}$, the cell population organizes in a nematic phase with a director at a finite angle with the stripe main direction. In parallel, cells spontaneously develop complex flows with shear and transverse components. Below the threshold $L_{\mathcal{c}}$, cells orient in the direction of the stripe and no net shear flow develops.

To understand these observations, we modeled our system as a confined active nematic fluid 27,28. Indeed, despite the obvious practical differences, our confined cells share the same fundamental symmetries as an acto-myosin network powered by ATP hydrolysis between parallel plates 29 . We therefore developed an adapted version of the physical model of Ref 29 which predicts that active nematics confined in a stripe of width $L$ exhibit a generic continuous transition at a critical width $L_{\mathcal{c}}$ between an ordered immobile state and a flowing state. This transition is analogous to the Fréedericksz transition of nematic liquid crystals 30 but it is driven by the intrinsic activity of the system rather than an external field. To account for the finite angle of the edge cells (Figure 2B), we adapted the model by assuming a finite anchoring at the walls. The torque exerted on the cells by the edges is then balanced by the nematic elastic torque (see Supplementary Note).

For widths larger than but close to the critical width $\left(L \gtrsim L_{\mathcal{c}}\right)$, the angle $\theta$ is predicted to vary across the stripe as $\theta(x) \sim \frac{\pi}{2}+\varepsilon \sqrt{\frac{L-L_{c}}{L_{c}}} \cos \left(q_{c} x\right)$ where $q_{c}=\frac{2 \pi}{L_{c}}$ and $\varepsilon$ is a coefficient that depends on the material properties of the system (see Supplementary Note). Similarly, the ycomponent of the velocity is given by $v_{y}(x) \sim \frac{2 K q_{c} \varepsilon}{\gamma(v+1)} \sqrt{\frac{L-L_{c}}{L_{c}}} \sin \left(q_{c} x\right)$, where $K$ is the stiffness associated to splay deformations of the director field, $\gamma$ is the rotational viscosity and $v$ is the flow alignment parameter. These quantities are material parameters of the active cells. Therefore, it is expected that the central angle $\theta(0)-\pi / 2$, the angle $\theta\left( \pm \frac{L}{2}\right)-\pi / 2$ at the edges, and the velocity at the edges $v_{y}\left( \pm \frac{L}{2}\right)$ all scale as $\sqrt{\left(L-L_{c}\right)}$ (see also Supplementary Note). We note that the large fluctuations of the orientations and velocities (Supplementary Fig. 7) are not described by this mean-field model. For this reason, we focus here on average quantities only.

Fitting model to experiments for $10 \mu \mathrm{m}<L<150 \mu \mathrm{m}$, we find a very good agreement between these theoretical scaling predictions and the experimental data yielding $L_{c}=46 \pm 4$ $\mu m$ for RPE1 cells (Figure 4A,B). Similar results were obtained for C2C12 mouse myoblasts. In this case, we measured $\lambda=12 \pm 2 \mu \mathrm{m}$ and $L_{c}=30 \pm 4 \mu \mathrm{m}$. The theory thus captures the main features of the biological system and in particular the existence of the transition that we now identify as a Fréedericksz transition driven by cell activity. Spontaneous flow transitions are typical of active matter; other examples include low- 
Reynolds number turbulence or spontaneous topological singularities in translation and rotation 27,31. Here, we demonstrate their importance in living tissues, which has potentially far-reaching consequences by strongly directing the motion of groups of cells over large distances.

However, some observations for $L>L_{c}$ could not be described by this simple model: Namely, i/ the $v_{y}(X)$ profile is screened at a finite length by friction on the surface (Figure 1H,J; Figure 2D,E; Supplementary Fig.6, 8), ii/ we observe transversal convergent flows toward the center of the stripe that are absent in the theory (Figure 1I,K; Figure 2F,G) and, iii/ in the experiments, the system exhibits a net chirality (Supplementary Fig. 4), i.e. the signs of the spontaneous shear flow and tilt angles are well-defined and not statistically distributed. These features can be accounted for by recognizing that $\mathrm{i} /$ momentum is not conserved in our experiments because of the interactions with the substrate, ii/ the cell number is not a conserved quantity since cells can divide and extrude, and iii/ many biological cellular systems have been reported to be chiral (Supplementary Note).

A finite cell-substrate friction is predicted to impact the velocity profile in the main direction of the stripe as we observe experimentally on the $v_{y}(x)$ profile (Supplementary Fig. 8). Large friction coefficients are expected to impair the development of the spontaneous shear flow and the associated tilt (Figure 4C, Supplementary Fig. 9). As a consequence, the behavior of the NIH-3T3 cells that fluctuate without generating any net shear flow and align with the stripe direction at all widths 13 (Figure 1E, Figure 2B,C, Supplementary Fig. 3), can be explained by either a lower activity of these cells or a larger cell-substrate friction.

The observation of transverse flows from the edges toward the center of the stripe is associated with a non-vanishing divergence of the velocity which is explained by a nonconservation of the cell number at steady state. The previously-observed larger number of cell extrusions in the central part of the confined monolayer compared to its edges 22 is the natural explanation for this observed convergent flow (Figure 4D). We note that the shear flow that originates from cell activity is not coupled to this convergent flow as blebbistatintreated RPE1 cells or untreated NIH-3T3 cells do not exhibit shear flow in spite of proliferation that results in convergent flow (Supplementary Fig. 5C, Supplementary Fig. $3 \mathrm{~J}, \mathrm{~K})$. Of notice, in the center of wide stripes, the shear flow is screened by friction and becomes very small (Figure 1J, Supplementary Fig. 6), while the convergent flow is not screened (Figure 1K). We find that the dynamics of the director's orientation is controlled by these convergent flows for stripes larger than typically $1000 \mu \mathrm{m}$ (see Supplementary Note, section 2).

Based on the left-right symmetry breaking observed in the experiments (Supplementary Fig. 4), we introduced in the hydrodynamic equations the chiral terms reflecting active mechanical and orientational torques, independently of the spontaneous flow transition 32,33 (Supplementary Note). This chiral contribution is the only finite term for $L<L_{c}$ and, as in any symmetry breaking transition, it selects only one of the two possible shear-flowing states for $L>L_{\mathcal{C}}$, therefore explaining the observed chirality. As a matter of fact, the small but finite fraction of experiments showing an opposite chirality confirms that the system is controlled by a mechanical instability. Another consequence of the existence of these chiral 
terms is to smoothen the variation of the velocity with $L$ at $L \sim \mathrm{L}_{\mathrm{c}}$ (Figure 4E). In the present situation, these chiral terms are sufficient to impose the chirality but too small to be experimentally measured from the analysis of the transition.

The coefficients used in the theory to fit the data are of the order of those routinely measured for cell populations (see Supplementary Note), hinting that this framework can be extended to other cell types and situations. Motivated by the in vivo observation of antiparallel flows of cancer cells having detached from the tumor and migrating collectively in confining effective channels 3, we confined HT1080 sarcoma cells in adhesive stripes. Indeed, these cells also developed a shear flow very similar to the two cell types studied in the present article although with an opposite chirality both regarding the tilt angle and the shear flow (Supplementary Fig. 10), highlighting the universality across cell lines of our conclusions.

In light of these in vitro results, we propose that the antiparallel cell displacements that contribute to cell transport in vivo in embryonic development or cancer may be the generic outcome of the confinement of the active nematic gels properties of these cell assemblies.

\section{Methods}

Cell culture

RPE1 cells (gift from Dr Maxime Dahan, Institut Curie), C2C12 myoblasts (gift from Dr Clotilde Théry, Institut Curie), HT1080 cells (gift from Dr Philippe Chavrier, Institut Curie) and NIH-3T3 fibroblasts (gift from Dr Karine Laud-Duval, Institut Curie) were cultured in Dulbecco's modified Eagle's medium (High glucose + GlutaMAX, Gibco) supplemented with 10\% FBS (Sigma) and 1\% antibiotics solution [penicillin (10,000 units $/ \mathrm{mL})+$ streptomycin $(10 \mathrm{mg} / \mathrm{mL})$; Gibco] at $37^{\circ} \mathrm{C}, 5 \% \mathrm{CO}_{2}$, and $90 \%$ humidity. Blebbistatin (Sigma) was used at a concentration of $3 \mu \mathrm{M}$ (higher concentrations destroyed the nematic order altogether).

\section{Time-lapse microscopy}

Time-lapse multifield experiments were performed in phase contrast on an automated inverted microscope (Olympus IX71) equipped with thermal and $\mathrm{CO}_{2}$ regulations. Typical field of view (FOV) was $1.5 \mathrm{~mm} \times 1.5 \mathrm{~mm}$. The displacements of the sample and the acquisitions with a CCD camera (Retiga 4000R, QImaging) were controlled by Metamorph (Universal Imaging) software. The typical delay between two successive images of the same field was set to 15 or 30 minutes.

\section{Image Processing}

Most of the image processing was performed using the ImageJ public domain software. The orientation field was obtained by computing the local structure tensor with ImageJ plugin OrientationJ 34,35. For representation purposes, we occasionally used a Line Integral Convolution (LIC) under Matlab (MathWorks). The velocity field was mapped by particle image velocimetry (PIV) analysis. Stacks of images were analyzed with a custom made PIV algorithm based on the MatPIV software package for MatLab (MathWorks Inc.) 7. The window size was set to 32 pixels $=23.75 \mu \mathrm{m}$ with a 0.5 overlap for $\mathrm{L}>30 \mu \mathrm{m}$ and 16 pixels $=$ 
$11.9 \mu \mathrm{m}$ with a 0.5 overlap for $\mathrm{L}=20 \mu \mathrm{m}$ and $\mathrm{L}=30 \mu \mathrm{m}$. The friction screening length $\lambda$ was measured by fitting two decreasing exponential curves to the $V_{y}$ velocity profiles.

\section{Micropatterning technique}

Clean glass substrates were first uniformly coated with a cell-repellent layer (interpenetrated gel of acrylamide and polyethylene glycol) 22. A photoresist mask was then structured directly on top of the layer by classical photolithography methods and air plasma was used to locally etch the protein-repellent coating through this mask. The photoresist was then removed with acetone yielding a cell repellant substrate where domains on which cells can adhere (bare glass) have been defined 22,36. Because of the frequent presence of topological defects at widths larger than $1500 \mu \mathrm{m}$, we have limited our study to stripe widths between $10 \mu \mathrm{m}$ and $1400 \mu \mathrm{m}$.

\section{Statistical analysis}

Statistical analysis was performed with Matlab (Mathworks) or Origin (Originlab). Experiments were performed in at least 2 replicas, each using 6 well plates with stripes of two distinct widths per well (for $\mathrm{L}=50 \mu \mathrm{m}, 100 \mu \mathrm{m}$ and $200 \mu \mathrm{m}$ to $1200 \mu \mathrm{m}$ ) or five distinct widths (for $\mathrm{L}=10$ to $200 \mu \mathrm{m}$ ). The number of analyzed FOVs for each width is reported in the list below. Error bars represent the SDs over all the FOVs analyzed (pooling all experiments in a single set). FOVs presenting topological defects were excluded from the analysis. The orientational profiles are all acquired $50 \mathrm{~h}$ after confluence. The velocity profiles obtained from the PIV velocity maps are averaged over time $(0<\mathrm{t}<50 \mathrm{~h}$ postconfluence). Both profiles were averaged along the direction of the stripe.

List of the widths $\mathrm{L}$ analyzed in the present study and the corresponding number $\mathrm{N}$ of FOVs used for the PIV analysis of RPE1 cells: $(\mathrm{L}(\mu \mathrm{m}), \mathrm{N})=(20,18) ;(30,21) ;(40,20) ;(50,56)$; $(60,21) ;(70,20) ;(80,29) ;(90,29) ;(100,61) ;(110,29) ;(120,29) ;(130,26) ;(140,26)$; (150, 26), (160, 26); (170, 35); (180, 35); (190, 35); (200, 60); (300, 20); (400, 13); (500, $13) ;(700,14) ;(800,11) ;(1000,18) ;(1200,7)$

List of the widths $\mathrm{L}$ analyzed in the present study and the corresponding number $\mathrm{N}$ of FOVs used for measuring the orientation profiles of RPE1 cells $(\mathrm{L}(\mu \mathrm{m}), \mathrm{N})=(10,13) ;(20,18) ;(30$, 21); (40, 20); (50,60); (60, 21); (70, 19); (80, 28); (90, 29); (100, 84); (110,29); (120, 29); $(130,26) ;(140,26) ;(150,26),(160,26) ;(170,35) ;(180,35) ;(190,35) ;(200,75) ;(300,28)$ $(400,51)(500,38)(700,17)(800,27)(1200,24)(1400,14)$.

List of the widths $\mathrm{L}$ analyzed in the present study and the corresponding number $\mathrm{N}$ of FOVs used for measuring the orientation profiles of $\mathrm{C} 2 \mathrm{C} 12$ cells $(\mathrm{L}(\mu \mathrm{m}), \mathrm{N})=(50,53)(100,52)$ $(200,80)(300,69)(400,88)(500,60)(600,64)(700,39)(800,23)(1000,29)(1200,36)$.

List of the widths $\mathrm{L}$ analyzed in the present study and the corresponding number $\mathrm{N}$ of FOVs used for the PIV analysis and estimation of Fréedericksz transition in C2C12 cells $(\mathrm{L}(\mu \mathrm{m})$, $\mathrm{N})=(16,6) ;(32,10) ;(40,11) ;(50,16) ;(65,8) ;(80,9) ;(95,6) ;(140,10)$. 
List of the widths $\mathrm{L}$ analyzed in the present study and the corresponding number $\mathrm{N}$ of FOVs used for measuring the orientation profiles of NIH-3T3 cells $(\mathrm{L}(\mu \mathrm{m}), \mathrm{N})=(50,125)$ $(150,100)(300,5)(400,67)(500,64)(550,44)(600,84)(800,96)(1000,82)(1200,98)$.

Middle angle distribution measurement and PIV analysis of HT1080 cells were obtained from 28 stripes of $125 \pm 5 \mu \mathrm{m}$ width in course of 25 hours, starting from confluency.

Blebbistatin treatment of RPE1 cells was performed 12 hours after the cells became confluent on $300 \mu \mathrm{m}$ wide stripes. PIV analysis was performed on 9 FOVs for 12 hours before and after the blebbistatin treatment.

\section{Supplementary Material}

Refer to Web version on PubMed Central for supplementary material.

\section{Acknowledgements}

It is a pleasure to thank the members of the Biology-inspired Physics at MesoScales (BiPMS) group and in particular Floriana Ascione, Trinish Sarkar and Hannah G. Yevick. We thank Leo Valon for suggesting the use of RPE1 cells. VY gratefully acknowledges the CelTisPhyBio Labex and the EU PRESTIGE program for financial support. GS is supported by the Francis Crick Institute which receives its core funding from Cancer Research UK (FC001317), the UK Medical Research Council (FC001317), and the Wellcome Trust (FC001317). The BiPMS group and the Physical Approach of Biological Problems group are members of the CelTisPhyBio Labex. The BiPMS group is a member of the Institut Pierre-Gilles de Gennes.

\section{References}

1. McLennan R, et al. Multiscale mechanisms of cell migration during development: theory and experiment. Development. 2012; 139:2935-2944. [PubMed: 22764050]

2. Clark AG, Vignjevic DM. Modes of cancer cell invasion and the role of the microenvironment. Curr Opin Cell Biol. 2015; 36:13-22. [PubMed: 26183445]

3. Weigelin B, Bakker G-J, Friedl P. Intravital third harmonic generation microscopy of collective melanoma cell invasion. IntraVital. 2012; 1:32-43. [PubMed: 29607252]

4. Trepat X, et al. Physical forces during collective cell migration. Nat Phys. 2009; 5:426-430.

5. Reffay M, et al. Interplay of RhoA and mechanical forces in collective cell migration driven by leader cells. Nat Cell Biol. 2014; 16:1-9.

6. Hakim V, Silberzan P. Collective cell migration: a physics perspective. Reports Prog Phys. 2017; 80:76601.

7. Petitjean L, et al. Velocity fields in a collectively migrating epithelium. Biophys J. 2010; 98:1790800. [PubMed: 20441742]

8. Londono $\mathrm{C}$, et al. Nonautonomous contact guidance signaling during collective cell migration. Proc Natl Acad Sci. 2014; 111:1807-1812. [PubMed: 24449852]

9. Garcia $S$, et al. Physics of active jamming during collective cellular motion in a monolayer. Proc Natl Acad Sci. 2015; 112:15314-15319. [PubMed: 26627719]

10. Sepúlveda N, et al. Collective Cell Motion in an Epithelial Sheet Can Be Quantitatively Described by a Stochastic Interacting Particle Model. PLoS Comput Biol. 2013; 9:e1002944. [PubMed: 23505356]

11. Basan M, Elgeti J, Hannezo E, Rappel W-J, Levine H. Alignment of cellular motility forces with tissue flow as a mechanism for efficient wound healing. Proc Natl Acad Sci. 2013; 110:24522459. [PubMed: 23345440]

12. Kabla AJ. Collective cell migration: leadership, invasion and segregation. J R Soc Interface. 2012; 9:3268-78. [PubMed: 22832363] 
13. Duclos G, Garcia S, Yevick HG, Silberzan P. Perfect nematic order in confined monolayers of spindle-shaped cells. Soft Matter. 2014; 10:2346-2353. [PubMed: 24623001]

14. Elsdale T. Parallel orientation of fibroblasts in vitro. Exp Cell Res. 1968; 51:439-450. [PubMed: 4300253]

15. Kemkemer R, Teichgräber V, Schrank-Kaufmann S, Kaufmann D, Gruler H. Nematic orderdisorder state transition in a liquid crystal analogue formed by oriented and migrating amoeboid cells. Eur Phys J E. 2000; 110:101-110.

16. Duclos G, Erlenkämper C, Joanny J-F, Silberzan P. Topological defects in confined populations of spindle-shaped cells. Nat Phys. 2016; 13:58-62.

17. Lois C, García-Verdugo JM, Alvarez-Buylla A. Chain migration of neuronal precursors. Science. 1996; 271:978-81. [PubMed: 8584933]

18. Nam SC, et al. Dynamic features of postnatal subventricular zone cell motility: A two-photon timelapse study. J Comp Neurol. 2007; 505:190-208. [PubMed: 17853439]

19. Wan LQ, et al. Micropatterned mammalian cells exhibit phenotype-specific left-right asymmetry. Proc Natl Acad Sci. 2011; 108:12295-12300. [PubMed: 21709270]

20. Raymond MJ, Ray P, Kaur G, Singh AV, Wan LQ. Cellular and Nuclear Alignment Analysis for Determining Epithelial Cell Chirality. Ann Biomed Eng. 2016; 44:1475-1486. [PubMed: 26294010]

21. Kawaguchi K, Kageyama R, Sano M. Topological defects control collective dynamics in neural progenitor cell cultures. Nature. 2017; 545:327-331. [PubMed: 28403137]

22. Deforet M, Hakim V, Yevick HG, Duclos G, Silberzan P. Emergence of collective modes and tridimensional structures from epithelial confinement. Nat Commun. 2014; 5:3747. [PubMed: 24796352]

23. Saw TB, et al. Topological defects in epithelia govern cell death and extrusion. Nature. 2017; 544:212-216. [PubMed: 28406198]

24. Chen T-H, et al. Left-Right Symmetry Breaking in Tissue Morphogenesis via Cytoskeletal Mechanics. Circ Res. 2012; 110:551-559. [PubMed: 22223355]

25. Tee $\mathrm{YH}$, et al. Cellular chirality arising from the self-organization of the actin cytoskeleton. Nat Cell Biol. 2015; 17:445-457. [PubMed: 25799062]

26. Xu J, et al. Polarity reveals intrinsic cell chirality. Proc Natl Acad Sci. 2007; 104:9296-9300. [PubMed: 17517645]

27. Marchetti MC, et al. Hydrodynamics of soft active matter. Rev Mod Phys. 2013; 85:1143-1189.

28. Kruse K, Joanny JF, Jülicher F, Prost J, Sekimoto K. Generic theory of active polar gels: a paradigm for cytoskeletal dynamics. Eur Phys J E. 2005; 16:5-16. [PubMed: 15688136]

29. Voituriez R, Joanny J-F, Prost J. Spontaneous flow transition in active polar gels. Europhys Lett. 2005; 70:404-410.

30. de Gennes, P-G, Prost, J. The physics of liquid crystals. 2nd Edition. Oxford University Press; 2003.

31. Prost J, Jülicher F, Joanny J-F. Active gel physics. Nat Phys. 2015; 11:111-117.

32. Fürthauer S, Strempel M, Grill SW, Jülicher F. Active chiral fluids. Eur Phys J E. 2012; 35:89. [PubMed: 23001784]

33. Fürthauer S, Strempel M, Grill SW, Jülicher F. Active Chiral Processes in Thin Films. Phys Rev Lett. 2013; 110:48103.

34. Rezakhaniha R, et al. Experimental investigation of collagen waviness and orientation in the arterial adventitia using confocal laser scanning microscopy. Biomech Model Mechanobiol. 2012; 11:461-473. [PubMed: 21744269]

35. Rasband, WS. ImageJ v1.46b. US National Institutes of Health; Bethesda, Maryland: 1997-2012. (Image J v1.46b (US National Institutes of Health, Bethesda, Maryland, 1997-2012)

36. Duclos, G, , et al. Cell Migration: Methods and Protocols, Methods in Molecular Biology. Gautreau, A, editor. Humana Press; New York: 2018. 387-399. 

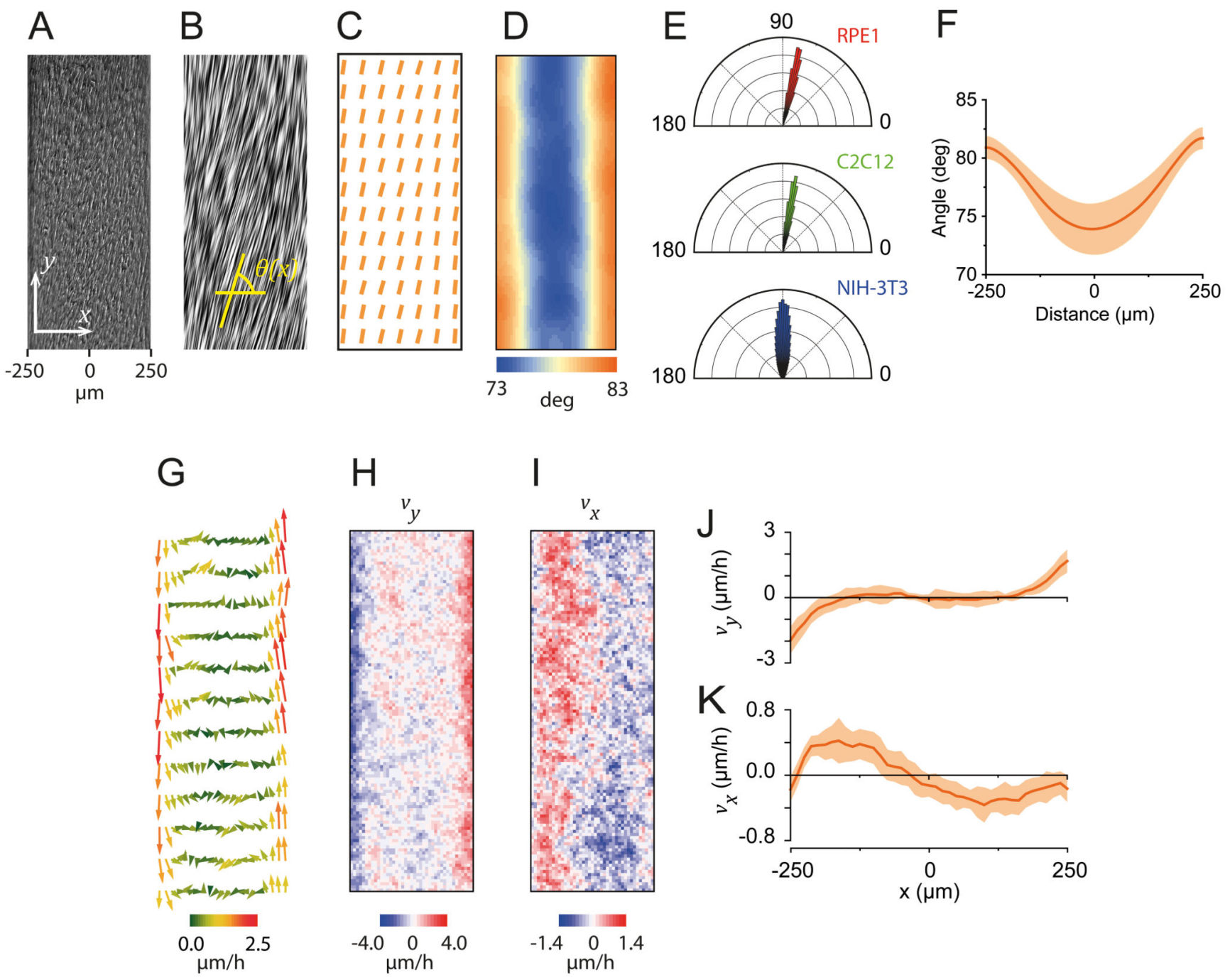

Figure 1. Confined RPE1 cells align together with a tilt angle and develop a shear flow.

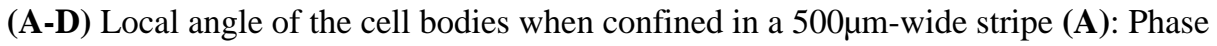
contrast; (B) Line integral convolution; (C) Local director. Only a small fraction of the directors is displayed for clarity; (D) Heatmap of the local angle. (E) Histograms of the angles $\theta$ of the cells' bodies (averaged over the width) $\left(\mathrm{n}_{\mathrm{RPE} 1}=38\right.$ FOVs, $\mathrm{n}_{\mathrm{C} 2 \mathrm{C} 12}=60 \mathrm{FOVs}$, $\mathrm{n}_{\mathrm{NIH}-3 \mathrm{~T} 3}=64$ FOVs). (F) Profile of the orientation angle across the stripe width after averaging along the y direction. (G) Velocity field within the stripe. The colors code for the speed. Only a fraction of the vectors is displayed for clarity. Note the shear flow (ycomponent) near the edges and the relatively smaller $\mathrm{x}$-component of the velocity directed toward the center. (H) and (I): Heatmaps of the $y$ - and $x$-components of the velocity. (J) and (K) Profiles of the two components of the velocity across the stripe after averaging over its length (y direction). For all relevant panels, solid lines are average values, colored areas are the SDs. 


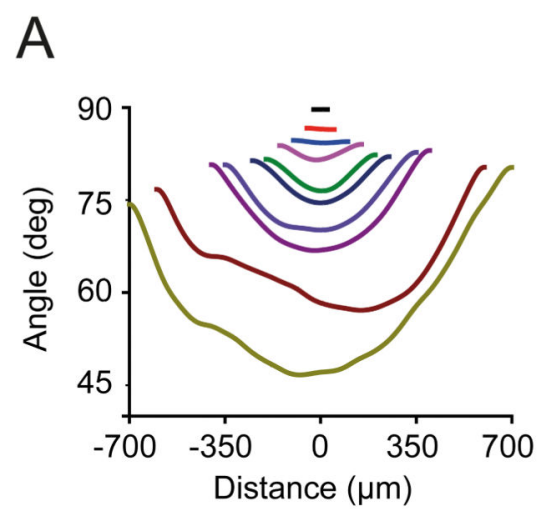

D

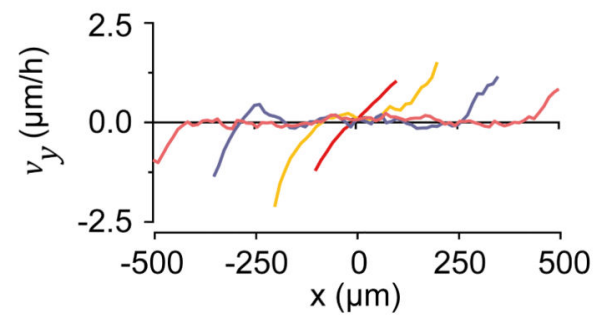

$\mathrm{F}$

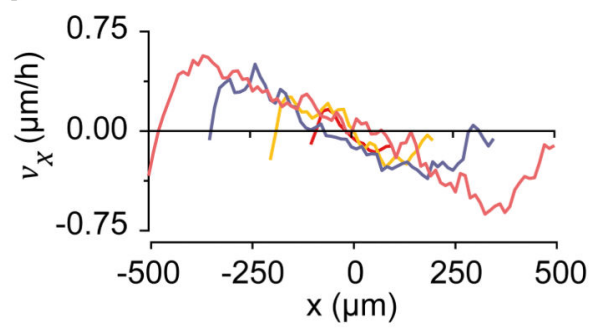

B

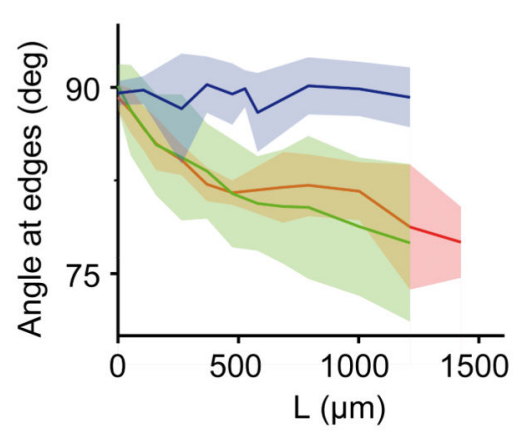

C

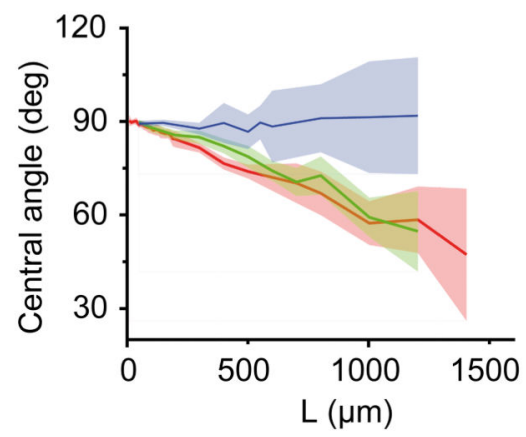

$\mathrm{E}$

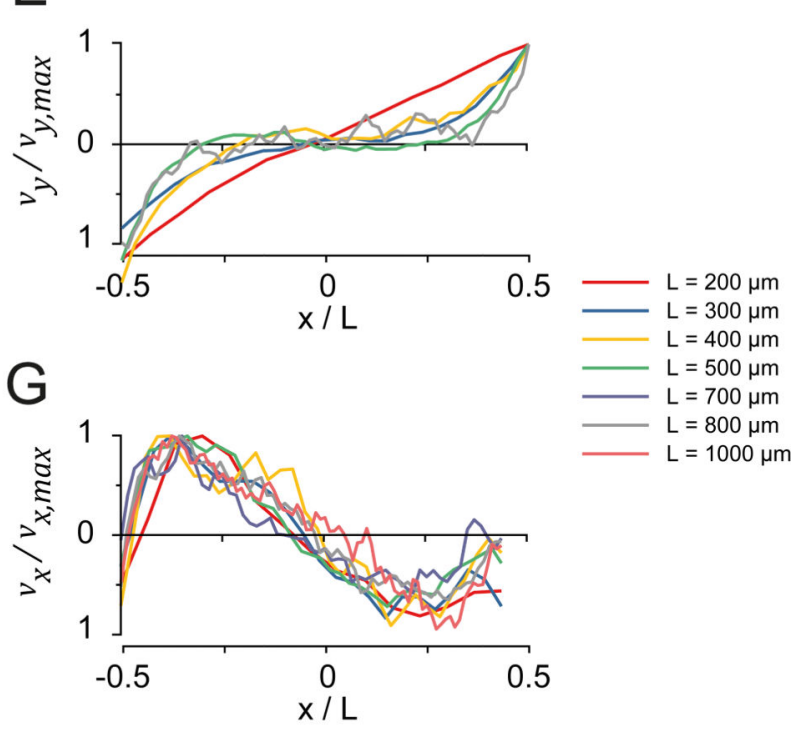

Figure 2. Influence of the width of the confining stripe on the behavior of the cells.

(A) Profiles of the angles across the stripe for different widths L. (B) and (C) evolution of the edge angle (B) and the central angle (C) of the cell body, as a function of the width. Blue NIH-3T3 cells, Red RPE1 cells, Green C2C12 cells. Lines are the average values, colored area are the SDs. (D) Profiles of the y-component of the velocity for different widths. (E) Rescaled y-component of the velocity as a function of the normalized width. Because of the friction with the substrate, this scaling does not result in a universal profile. (F) Profiles of the x-component of the velocity for different widths. (G) Rescaled x-component of the velocity as a function of the normalized width. In this case, this scaling results in a universal profile. All experiments performed with RPE1 cells except when indicated. 

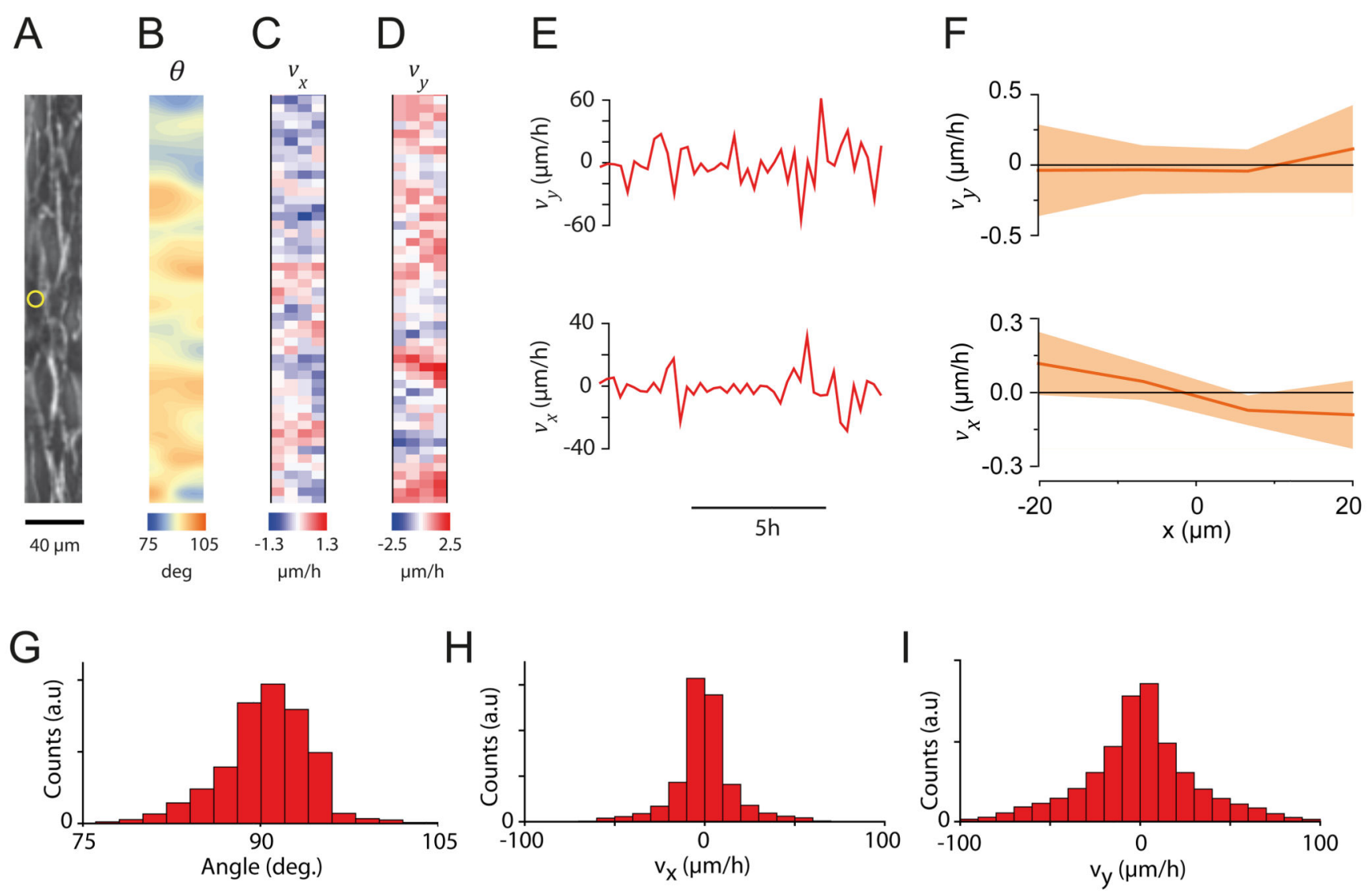

Figure 3. Below a critical width, the cells orient along the stripe and don't flow in average.

(A) Phase contrast image of the RPE1 cells in a $40 \mu \mathrm{m}$-wide stripe. (B) The directions of the cell bodies fluctuate in space about a $90^{\circ}$ value. (C) and (D) The $\mathrm{x}$ - and y-components of the velocity fluctuate in space about a zero value. (E) Time traces of the $\mathrm{x}$ - and $\mathrm{y}$ components of the velocity. Measurements taken at the edge of the stripe (see yellow circle on (A)). The cells are motile but fluctuate about their mean position with no net displacement. (F) Profiles if the two components of the velocity across the stripe. Note the absence of shear flow $\left(\mathrm{v}_{\mathrm{y}}=0\right)$ while the convergent flow is maintained (solid lines are average values, colored areas are the SDs; $\mathrm{n}=18$ FOVs). (G-I) Distributions of the angle, $\mathrm{x}-$ and y-components of the velocity. $\theta=90^{\circ} \pm 4^{\circ} ; v_{x}=0 \pm 16 \mu \mathrm{m} / \mathrm{h} ; \mathrm{v}_{\mathrm{y}}=0 \pm 33 \mu \mathrm{m} / \mathrm{h} . \mathrm{N}=20$ independent stripes. 
A
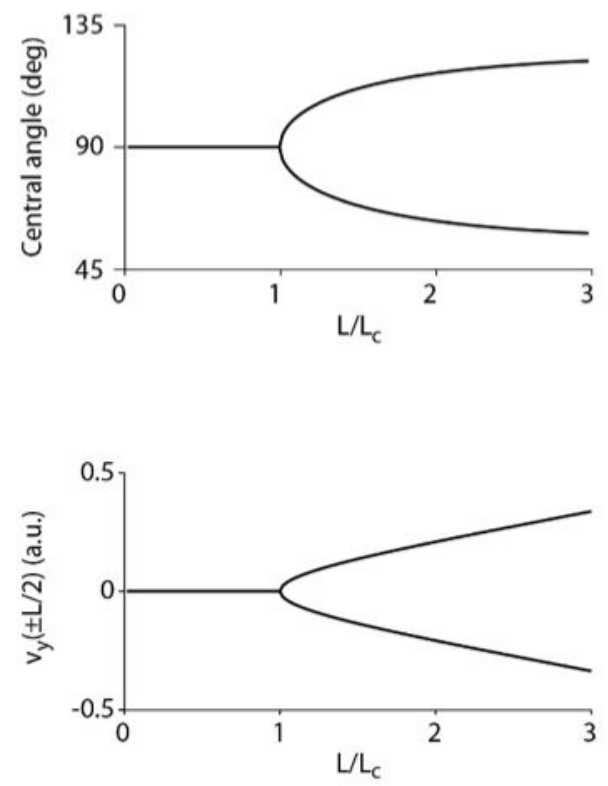

B
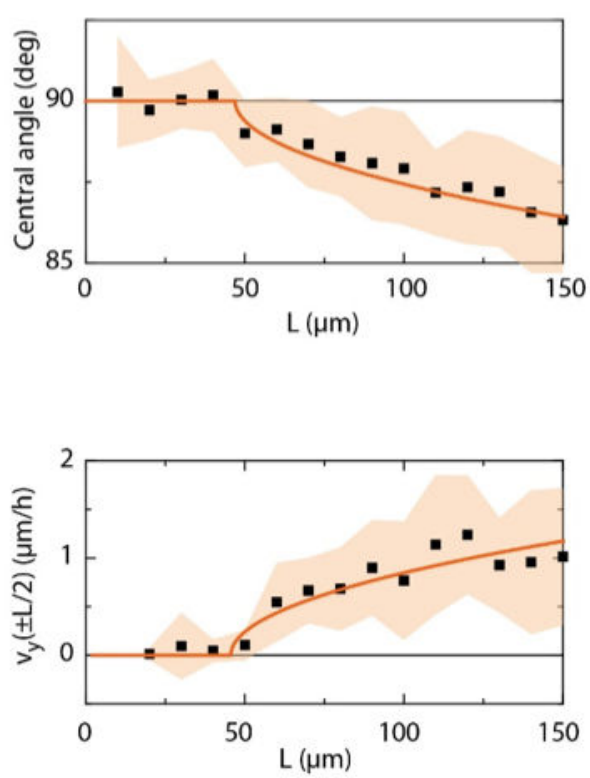

C

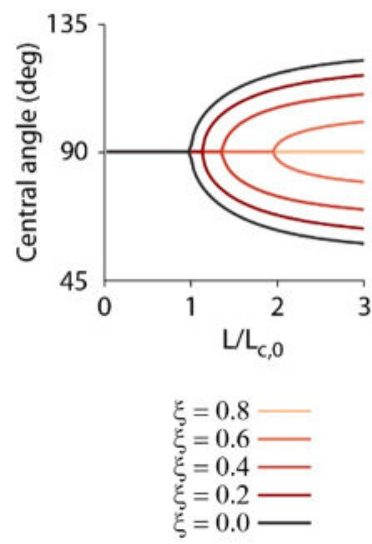

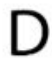

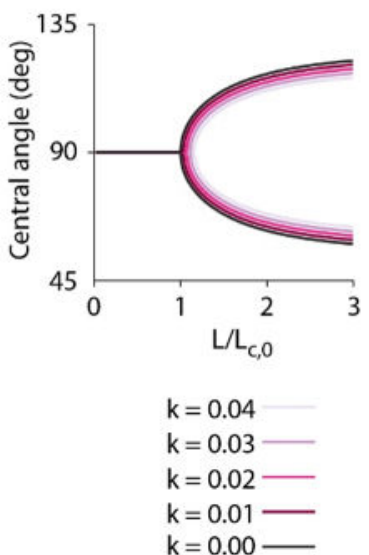

E

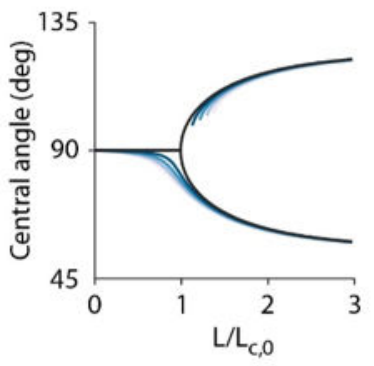

$\zeta_{\mathrm{PC}}=0.04$

$\zeta_{\mathrm{PC}}=0.03$

$\zeta_{\mathrm{PC}}=0.02$

$\zeta_{\mathrm{PC}}=0.01$

$\zeta_{\mathrm{PC}}=0.00$

Figure 4. Comparison of the experimental results with the active gel theory.

(A) Theoretical prediction of the evolution of the central angle and shear component of the velocity as a function of the width. Width has been rescaled by the critical width. In this basic version of the model, friction, chirality and convergent flows are not considered. (B) ycomponent of the velocity and central angle as a function of the stripe width. The points are the experimental data (colored areas are the SDs) and the lines are the theoretical fits by a function $\sqrt{L-L_{c}}$ for $L>L_{c}$. Note the difference of horizontal scale with Figures 2B,C as we focus here on the close vicinity of the critical width. (C)-(E) Impact of the friction (C), the transverse flows (D) and the chirality (E) on the transition. The widths have been rescaled by the critical width. Note the modest impact of transverse flow on the transition and the non- 
symmetric contribution of the chiral term that selects one branch and smoothen out the transition. The parameters have been set to $K=\gamma=\zeta \Delta \mu=\eta=-\frac{v}{2}=W_{S}=1$; and $\xi=k=\zeta_{P C} \Delta \mu=\lambda_{P C}=0$ as default values (see Supplementary Note for definitions). 